\title{
PROJECTIVE SCHUR FUNCTIONS AS A BISPHERICAL FUNCTIONS ON CERTAIN HOMOGENEOUS SUPERSPACES
}

\author{
ALEXANDER SERGEEV
}

\begin{abstract}
I show that the projective Schur functions may be interpreted as bispherical functions of either the triple $(\mathfrak{q}(n), \mathfrak{q}(n) \oplus \mathfrak{q}(n), \mathfrak{q}(n))$, where $\mathfrak{q}(n)$ is the "odd" (queer) analog of the general linear Lie algebra, or the triple $(\mathfrak{p} \mathfrak{e}(n), \mathfrak{g l}(n \mid n), \mathfrak{p e}(n))$, where $\mathfrak{p e}(n)$ is the periplectic Lie superalgebra which preserves the nondegenerate odd bilinear form (either symmetric or skew-symmetric). Making use of this interpretation I characterize projective Schur functions as common eigenfunctions of an algebra of differential operators.
\end{abstract}

\section{INTRODUCTION}

The ground field is $\mathbb{C}$.

1.1. In [Sch] I. Schur introduced projective Schur functions as characteristics of projective representations of symmetric groups. In [Se1] I showed that these characteristics are actually characters of tensor representations of Lie superalgebra $\mathfrak{q}(n)$.

In [St] Stembridge interpreted projective Schur functions as characteristics of of spherical functions of a certain twisted Gelfand pair (for definition see [St]). Here I will remind definition of bispherical functions and demonstrate that these Stembridge's characteristics are precisely bispherical functions of the triple $(\mathfrak{p e}(n), \mathfrak{g l}(n \mid n), \mathfrak{p e}(n))$, where $\mathfrak{p e}(n)$ can be embedded into $\mathfrak{g l}(n \mid n)$ in two different ways (corresponding to interpretation of $\mathfrak{p e}(n)$ as the algebra preserving a symmetric odd bilinear form or a skew-symmetric one).

One obtains one more realization of projective Schur functions if one considers bispherical functions of the triple $(\mathfrak{q}(n), \mathfrak{q}(n) \oplus \mathfrak{q}(n), \mathfrak{q}(n))$, where $\mathfrak{q}(n)$ can be embedded into $\mathfrak{q}(n) \oplus \mathfrak{q}(n)$ by one of the two ways: either as the diagonal or as a "twisted diagonal".

Both ways to realize projective Schur functions allow one to construct an algebra of differential operators for which the projective Schur functions are eigenfunctions. This algebra appears as the algebra of radial parts of Laplace operators for the Lie superalgebra $\mathfrak{g l}(n \mid n)$ or $\mathfrak{q}(n)$.

1.2. Differential operators and projective Schur functions. Let $I=\{1, \ldots, n\}, V$ an $n$-dimensional vector space, $\left\{e_{i}\right\}_{i \in I}$ a basis of $V$, and $\left\{\varepsilon_{i}\right\}$ the dual basis of $V^{*}$. If $l \in V^{*}$, than $e^{l}$ denotes a homomorphism $S(V) \longrightarrow \mathbb{C}$, where $S(V)$ is the symmetric algebra of $V$. Recall that $S(V)^{*}$ can be identified with the algebra of formal power series in $n$ indeterminates.

On $S(V)^{*}$, define a family of differential operators. Set $\varepsilon_{i j}=\varepsilon_{i}-\varepsilon_{j}$ for $i, j \in I, i \neq j$ and set

$$
\partial_{i}^{(1)}\left(e^{l}\right)=l\left(e_{i}\right) e^{l}=\partial_{i}\left(e^{l}\right) \text { for any } l \in V^{*},
$$

1991 Mathematics Subject Classification. 05E05, $17 \mathrm{~B} 35$.

Key words and phrases. Schur functions, Lie superalgebras, homogeneous superspaces.

I am thankful to G. Olshanskii who raised the problem, D. Leites for help and Isaac Newton Institute for hospitality and support. 
set further

$$
\partial_{i}^{(k)}= \begin{cases}\partial_{i} \partial_{i}^{(k-1)}+\sum_{j \neq i} \frac{2}{e^{\varepsilon_{i j}}-e^{\varepsilon_{j i}}}\left(\partial_{i}^{(k-1)}-\partial_{j}^{(k-1)}\right) & \text { for } k \text { odd }, \\ \left(\partial_{i}-1\right) \partial_{i}^{(k-1)}+\sum_{j \neq i}\left(\frac{2}{e^{\varepsilon_{i j}}-e^{\varepsilon_{j i}}} \partial_{i}^{(k-1)}-\frac{2 e^{\varepsilon_{i j}}}{e^{\varepsilon_{i j}}-e^{\varepsilon_{j i}}} \partial_{i}^{(k-1)}\right) & \text { for } k \text { even } .\end{cases}
$$

Finally, for $k$ odd, set

It is not difficult to verify directly that

$$
\Omega_{k}=\sum_{i=1}^{n} \partial_{i}^{(k)} .
$$

$$
\begin{aligned}
& \Omega_{3}=\sum_{1}^{n} \partial_{i}^{3}+\sum_{i<j} \frac{6}{e^{\varepsilon_{i j}}-e^{\varepsilon_{j i}}}\left(\partial_{i}^{2}-\partial_{j}^{2}\right)-\sum \frac{6}{\left(e^{\frac{1}{2} \varepsilon_{i j}}+e^{\frac{1}{2} \varepsilon_{j i}}\right)^{2}}\left(\partial_{i}+\partial_{j}\right) \\
& +24 \sum_{i \notin\{j, k\}} \frac{1}{\left(e^{\varepsilon_{i j}}-e^{\varepsilon_{j i}}\right)\left(e^{\varepsilon_{i k}}-e^{\varepsilon_{k i}}\right)} \partial_{i}-\left(\sum_{1}^{n} \partial_{i}\right)^{2},
\end{aligned}
$$

where $\{j, k\} \subset I$ is any two-element subset.

Introduce new indeterminates: $x_{i}=e^{\varepsilon_{i}}$. Then $\partial_{i}=x_{i} \frac{\partial}{\partial x_{i}}$ and

$$
\partial_{i}^{(k)}= \begin{cases}\partial_{i} \partial_{i}^{(k-1)}+\sum_{j \neq i} \frac{2 x_{i} x_{j}}{x_{i}^{2}-x_{j}^{2}}\left(\partial_{i}^{(k-1)}-\partial_{j}^{(k-1)}\right) & \text { for } k \text { odd } \\ \left(\partial_{i}-1\right) \partial_{i}^{(k-1)}+\sum_{j \neq i}\left(\frac{2 x_{i} x_{j}}{x_{i}^{2}-x_{j}^{2}} \partial_{i}^{(k-1)}-\frac{2 x_{i}^{2}}{x_{i}^{2}-x_{j}^{2}} \partial_{j}^{(k-1)}\right) & \text { for } k \text { even } .\end{cases}
$$

We have

$$
\begin{aligned}
& \Omega_{3}=\sum \partial_{i}^{3}+6 \sum_{i<j} \frac{x_{i} x_{j}}{x_{i}^{2}-x_{j}^{2}}\left(\partial_{i}^{2}-\partial_{j}^{2}\right)-6 \sum_{i<j} \frac{x_{i} x_{j}}{\left(x_{i}+x_{j}\right)^{2}}\left(\partial_{i}+\partial_{j}\right) \\
& +24 \sum_{i<j<k} \frac{x_{i} x_{j} x_{k}}{\left(x_{i}^{2}-x_{j}^{2}\right)\left(x_{i}^{2}-x_{k}^{2}\right)}\left(x_{i}\left(x_{j}^{2}-x_{k}^{2}\right) \partial_{i}-x_{j}\left(x_{i}^{2}-x_{k}^{2}\right) \partial_{j}+x_{k}\left(x_{i}^{2}-x_{j}^{2}\right) \partial_{k}\right) \\
& -\left(\sum \partial_{i}\right)^{2} .
\end{aligned}
$$

For $i=1, \ldots, n$ and any $k$, define differential operators $\tilde{\partial}_{i}^{(k)}$ and $\tilde{\partial}_{\bar{\imath}}^{(k)}$ by setting

$$
\tilde{\partial}_{i}^{(1)}=\tilde{\partial}_{i}=\tilde{\partial}_{\bar{\imath}}^{(1)}=\tilde{\partial}_{\bar{\imath}}=\partial_{i}
$$

and

$$
\begin{aligned}
\tilde{\partial}_{i}^{(k)} & =\tilde{\partial}_{i} \tilde{\partial}_{i}^{(k-1)}+\sum_{j \neq i} \frac{e^{\frac{1}{2} \varepsilon_{i j}}}{e^{\frac{1}{2} \varepsilon_{i j}}-e^{\frac{1}{2} \varepsilon_{j i}}}\left(\tilde{\partial}_{i}^{(k-1)}-\tilde{\partial}_{j}^{(k-1)}\right) \\
& -\sum_{j \neq i} \frac{e^{\frac{1}{2} \varepsilon_{i j}}}{e^{\frac{1}{2} \varepsilon_{i j}}+e^{\frac{1}{2} \varepsilon_{j i}}}\left(\tilde{\partial}_{i}^{(k-1)}+\tilde{\partial}_{\bar{\jmath}}^{(k-1)}\right), \\
\tilde{\partial}_{\bar{\imath}}^{(k)} & =-\tilde{\partial}_{i} \tilde{\partial}_{\bar{\imath}}^{(k-1)}-\sum_{j \neq i} \frac{e^{\frac{1}{2} \varepsilon_{i j}}}{e^{\frac{1}{2} \varepsilon_{i j}}-e^{\frac{1}{2} \varepsilon_{j i}}}\left(\tilde{\partial}_{\bar{\imath}}^{(k-1)}-\tilde{\partial}_{\bar{\jmath}}^{(k-1)}\right) \\
& +\sum \frac{e^{\frac{1}{2} \varepsilon_{i j}}}{e^{\frac{1}{2} \varepsilon_{i j}}+e^{\frac{1}{2} \varepsilon_{j i}}}\left(\tilde{\partial}_{\bar{\imath}}^{(k-1)}+\tilde{\partial}_{j}^{(k-1)}\right) .
\end{aligned}
$$

Set further

$$
\widetilde{\Omega}_{k}=\sum_{i=1}^{n}\left(\tilde{\partial}_{i}^{(k)}+\tilde{\partial}_{\bar{\imath}}^{(k)}\right) .
$$


1.2.1. Lemma . The algebra $\Omega$ generated by operators $\Omega_{k}$ for $k=1,3,5, \ldots$ coincides with the algebra generated by operators $\widetilde{\Omega}_{k}$ for $k=1,2,3,4, \ldots$.

1.2.2. Let $x_{1}, \ldots, x_{n}, t$ be indeterminates. Introduce polynomials $q_{k}\left(x_{1}, \ldots, x_{n}\right)$ from equation

$$
\sum_{k=0}^{\infty} q_{k}\left(x_{1}, \ldots, x_{n}\right) t^{k}=\frac{\prod\left(1+x_{i} t\right)}{\prod\left(1-x_{i} t\right)}
$$

Further, set

$$
Q_{k, l}=q_{k} q_{l}+2 \sum_{p=1}^{l} q_{k+p} q_{l-p} .
$$

We see that $Q_{(k, 0)}=q_{k}$ and $Q_{k, l}=-Q_{l, k}$ for $k+l>0$. If $\lambda=\left(\lambda_{1}, \ldots, \lambda_{m}\right)$ is a strict partition, i.e., $\lambda_{1}>\lambda_{2}>\ldots>\lambda_{m}>0$, then $(P f$ is the Pfaffian of the skew-symmetric matrix )

$$
Q_{\lambda}\left(x_{1}, \ldots, x_{n}\right)= \begin{cases}\operatorname{Pf}\left(Q_{\lambda_{i} \lambda_{j}}\right) & \text { if } m \text { is even } \\ Q_{(\lambda, 0)} & \text { if } m \text { is odd }\end{cases}
$$

1.2.3. Lemma . Set $\delta=\prod_{1 \leq i<j \leq n}\left(\frac{e^{\frac{1}{2} \varepsilon_{i j}}+e^{\frac{1}{2} \varepsilon_{j i}}}{e^{\frac{1}{2} \varepsilon_{i j}}-e^{\frac{1}{2} \varepsilon_{j i}}}\right)$, then

i) $\delta^{-1} \Omega_{3} \delta=\sum_{1}^{n} \partial_{i}^{3}-\left(\sum_{1}^{n} \partial_{i}\right)^{2}$.

ii) The polynomials $Q_{\lambda}$ are common eigenfunctions of the operators $\Omega_{k}$ for $k=1,3,5, \ldots$.

iii) Let $P\left(x_{1}, \ldots, x_{n}\right)$ be polynomial symmetric with respect to $x_{1}, \ldots, x_{n}$ and such that after substitution $x_{i}=t, x_{j}=-t$ it becomes independent of $t$. If $P$ is an eigenfunction of all the operators $\Omega_{k}, k=1,3,5, \ldots$, then, up to a scalar multiple, $P$ coinsides with one of the $Q_{\lambda}$.

1.3. Bispherical functions. Let $\mathfrak{g}$ be a finite dimensional Lie superalgebra. Its enveloping algebra $U(\mathfrak{g})$ possesses a canonical antiautomorphism $t: u \mapsto{ }^{t} u$ which extends the principal antiautomorphism of $\mathfrak{g}$ given by the formula $t(x)=-x$ for any $x \in \mathfrak{g}$ as follows:

$$
{ }^{t}(u v)=(-1)^{p(u) p(v) t}(v)^{t}(u) .
$$

Convention . For brevity, when it can not cause misunderstanding I sometimes write $(-1)^{u}$ instead of $(-1)^{p(u)}$. So $(-1)^{u+\overline{1}}=(-1)^{p(u)+1}$ is not the same as $(-1)^{p(u+1)}$.

The left and right coregular representations of $U(\mathfrak{g})$ are defined for any $l \in U(\mathfrak{g})^{*}$ and $v, u \in U(\mathfrak{g})$ by the formulas

$$
\left(L^{*}(u) l\right)(v)=(-1)^{u l} l\left(u^{t} \cdot v\right) \text { and }\left(R^{*}(u) l\right)(v)=(-1)^{u(l+v)} l(v u) .
$$

Let $\mathfrak{b}_{1}$ and $\mathfrak{b}_{2}$ be subalgebras of $\mathfrak{g}$. A functional $l \in U(\mathfrak{g})^{*}$ is called two-side invariant if

$$
l\left(x_{1} u\right)=l\left(u x_{2}\right)=0 \text { for any } x_{1} \in \mathfrak{b}_{1}, x_{2} \in \mathfrak{b}_{2} \text { and } u \in U(\mathfrak{g}) .
$$

Let $V$ be a $\mathfrak{g}$-module containing a nonzero $\mathfrak{b}_{2}$-invariant vector $v \in V$; suppose also that there exists a $\mathfrak{b}_{1}$-invariant vector $v^{*} \in V^{*}$. The matrix coefficient $\Theta\left(v^{*}, v\right) \in U(\mathfrak{g})^{*}$ defined by the formula

$$
\Theta\left(v^{*}, v\right)(u)=(-1)^{u v} v^{*}(u v) \text { for } u \in U(\mathfrak{g})
$$

is a bispherical function associated with the triple $\left(V, v^{*}, v\right)$.

Observe that if $z \in Z(\mathfrak{g})$, then $L^{*}(z) l$ is two-side invariant if so is $l$. Therefore, on the space of invariant functionals, every $z \in Z(\mathfrak{g})$ determines a linear operator $\Omega_{(z)}: l \mapsto L^{*}(z) l$. 
1.4. In addition to the usual $\mathfrak{g}$-module structure on $U(\mathfrak{g})$, the extention of the adjoint action, consider the $\mathfrak{g}$-module structure on $U(\mathfrak{g})$ with respect to the following action ([Se4, [G])

$$
x * u=x u-(-1)^{x(u+\overline{1})} u x .
$$

It is easy to verify that for a finite dimensional $\mathfrak{g}$-module $V$ the functional $u \mapsto \operatorname{tr}_{V}(u)$ is invariant with respect to this action.

In $\mathfrak{g} \oplus \mathfrak{g}$, consider two subalgebras:

$$
\mathfrak{g}_{1}=\left\{\left(x,(-1)^{x} x\right) \mid x \in \mathfrak{g}\right\} \text { and } \mathfrak{g}_{2}=\{(x, x) \mid x \in \mathfrak{g}\} .
$$

Lemma . The algebra of functionals on $U(\mathfrak{g} \oplus \mathfrak{g})$ biinvariant with respect to $\mathfrak{g}_{1}$ and $\mathfrak{g}_{2}$ is isomorphic to the algebra of functionals on $U(\mathfrak{g})$ invariant with respect to the action (1.4.1).

1.4.1. Convention . Let $I=I_{\overline{0}} \cup I_{\overline{1}}=\{1, \ldots, n\} \cup\{\overline{1}, \ldots, \bar{n}\}$ be the union of the "even" and "odd" indices. Let $\operatorname{dim} V=(n \mid n)$ and $\left\{e_{i}\right\}_{i \in I}$ a basis of $V$ such that the parity of each vector of the basis is the same as that of its index. We assume that $\overline{\bar{i}}=i$ and define the odd operator $\Pi \in \mathfrak{g l}(V)$ by setting

$$
\Pi\left(e_{i}\right)=(-1)^{p(i)} e_{\bar{i}}, \text { for any } i \in I .
$$

Define the parity operator $P \in \mathfrak{g l}(V)$ by setting

$$
P\left(e_{i}\right)=(-1)^{p(i)} e_{i} \text {, for any } i \in I .
$$

We denote the superspace of operators in $V$ by $\operatorname{End}(V)$, the superspace of matrices (in the standard format with respect to the basis in which all the even vectors come first) by $\operatorname{Mat}(V)$ and the Lie superalgebra structure in these isomorphic superspaces is denoted by $\mathfrak{g l}(V)$ or by $\mathfrak{g l}(\operatorname{dim} V)$.

\subsubsection{Queer superalgebras $\mathfrak{q}(V)$. Let}

$$
\mathfrak{q}(n)=\{X \in \mathfrak{g l}(V) \mid[X, \Pi]=0\} .
$$

It is easy to verify that $\mathfrak{q}(n)=\operatorname{Span}\left(e_{i j}, f_{i j} \mid i, j \in I_{\overline{0}}\right)$, where

$$
e_{i j}=e_{i} \otimes e_{j}+e_{\bar{i}} \otimes e_{\bar{\jmath}}, \quad f_{i j}=e_{i} \otimes e_{\bar{j}}^{*}+e_{\bar{i}} \otimes e_{j}^{*}, \text { for any } i, j \in I_{\overline{0}}
$$

and where $\left\{e_{i}^{*}\right\}_{i \in I}$ is the left dual of the basis $\left\{e_{i}\right\}_{i \in I}$; we have also identified $\operatorname{End}(V)$ with $V \otimes V^{*}$.

Set $\mathfrak{g}=\mathfrak{q}(n) \oplus \mathfrak{q}(n)$ and select $\mathfrak{g}_{1}$ and $\mathfrak{g}_{2}$ as at the beginning of sec. 1.4. Let $\sigma: \mathfrak{q}(n) \longrightarrow \mathfrak{g}$ be the embedding into the first summand, $\mathfrak{h}_{\overline{0}}=\operatorname{Span}\left(e_{i i} \mid i \in I_{\overline{0}}\right)$ be the even part of Cartan subalgebra of $\mathfrak{q}(n)$. Let us define inductively the following elements of $U(\mathfrak{q}(n))$ :

$$
\begin{gathered}
e_{i j}^{(1)}=e_{i j}, f_{i j}^{(1)}=f_{i j} \\
e_{i j}^{(p)}=\sum_{l=1}^{n} e_{i l} e_{l j}^{(p-1)}+(-1)^{k-1} \sum_{l=1}^{n} f_{i l} f_{l j}^{(p-1)} \\
f_{i j}^{(p)}=\sum_{l=1}^{n} e_{i l} f_{l j}^{(p-1)}+(-1)^{k-1} \sum_{l=1}^{n} f_{i l} e_{l j}^{(p-1)} .
\end{gathered}
$$


The following relations are subject to straightforward verification:

$$
\begin{aligned}
{\left[e_{i j}, e_{k l}^{(p)}\right] } & =\delta_{j k} e_{i l}^{(p)}-\delta_{i l} e_{k j}^{(p)}, \\
{\left[e_{i j}, f_{k l}^{(p)}\right] } & =\delta_{j k} f_{i l}^{(p)}-\delta_{i l} f_{k j}^{(p)}, \\
{\left[f_{i j}, e_{k l}^{(p)}\right] } & =(-1)^{p+1} \delta_{j k} f_{i l}^{(p)}-\delta_{i l} f_{k j}^{(p)}, \\
{\left[f_{i j}, f_{k l}^{(p)}\right] } & =(-1)^{p+1} \delta_{j k} e_{i l}^{(p)}+\delta_{i l} e_{k j}^{(p)} .
\end{aligned}
$$

As is not difficult to verify, see [Se3], the elements $z_{k}=\sum_{i=1}^{n} e_{i i}^{(k)}, k=1,3,5, \ldots$, are central ones if we embed $\mathfrak{q}(n)$ into $\mathfrak{g}=\mathfrak{q}(n) \oplus \mathfrak{q}(n)$ as the first summand, i.e., strictly speaking, I mean not $z_{k}$ but $\sigma\left(z_{k}\right) \in U(\mathfrak{g})$.

Let $\left\{\varepsilon_{i}\right\}_{i \in I_{\overline{0}}}$ be the basis of $\mathfrak{h}_{\overline{0}}$ dual to $\left\{e_{i}\right\}_{i \in I_{\overline{0}}}$. Let $\lambda=\left(\lambda_{1}, \ldots, \lambda_{m}\right)$ be a strict partition of $k$, i.e., $\lambda_{1}>\lambda_{2}>\ldots>\lambda_{m}>0$. Let $V^{\lambda}$ be an irreducible submodule of $V^{\otimes k}$ corresponding to $\lambda$, see [Se1]. Then the $\mathfrak{g}$-module $w^{\lambda}=V^{\lambda} \otimes\left(V^{\lambda}\right)$ is irreducible and contains a unique even $\mathfrak{g}_{2^{-}}$ invariant vector $\omega_{\lambda}$ corresponding to the identity operator under identification $V^{\lambda} \otimes\left(V^{\lambda}\right)^{*}=$ $\operatorname{End}\left(V^{\lambda}\right)$. The dual module $\left(V^{\lambda}\right)^{*} \otimes V^{\lambda}$ is also irreducible and contains an even $\mathfrak{g}_{1}$-invariant vector $\omega_{\lambda}^{*}$ corresponding to the parity operator $P$ under identification $\left(V^{\lambda}\right)^{*} \otimes V^{\lambda}=\operatorname{End}\left(V^{\lambda}\right)$.

1.5. Theorem . Let $\mathfrak{q}(n)$ be embedded into $\mathfrak{g}=\mathfrak{q}(n) \oplus \mathfrak{q}(n)$ as the first summand. Then

i) Each two-side invariant with respect to $\mathfrak{g}_{1}$ and $\mathfrak{g}_{2}$ functional on $\mathfrak{g}$ is uniquely determined by its restriction onto $S\left(\mathfrak{h}_{\overline{0}}\right) \subset \mathfrak{q}(n)$.

ii) Every $z \in Z(\mathfrak{q}(n))$ uniquely determines a differential operator $\Omega_{(z)}$ on the space of restrictions of left-invariant functionals $S\left(\mathfrak{h}_{\overline{0}}\right)^{* i n v}$.

iii) $\Omega_{\left(z_{k}\right)}=\Omega_{k}$ for $z=z_{k}$.

iv) Let $\varphi_{\lambda}=\Theta\left(\omega_{\lambda}^{*}, \omega_{\lambda}\right)$. Then the restriction of $\varphi_{\lambda}$ onto $S\left(\mathfrak{h}_{\overline{0}}\right)$ coincides up to a scalar multiple with $Q_{\lambda}\left(e^{\varepsilon_{1}}, \ldots, e^{\varepsilon_{n}}\right)$

1.6. Let $\mathfrak{g}=\mathfrak{g l}(\operatorname{dim} V)$, where $\operatorname{dim} V=(n \mid n)$. Let $I=I_{\overline{0}} \cup I_{\overline{1}}$, where $I_{\overline{0}}=\{1, \ldots, n\}$ and $I_{\overline{1}}=\{\overline{1}, \ldots, \bar{n}\}$. Let $\left\{e_{i}\right\}$ be a basis of $V$ such that the parity of each vector coincides with that of its index. Denote by $\mathfrak{p e}_{1}(n)$ and $\mathfrak{p e}_{2}(n)$ the Lie subsuperalgebras in $\mathfrak{g}$ preserving the respective tensors:

$$
\sum_{i \in I} e_{i}^{*} \otimes e_{i}^{*}, \quad \sum_{i \in I}(-1)^{i} e_{i}^{*} \otimes e_{i}^{*} .
$$

Let $\psi_{1}$ and $\psi_{2}$ be involutive antiautomorphisms of $\mathfrak{g}$ that single out $\mathfrak{p e}_{1}(n)$ and $\mathfrak{p e}_{2}(n)$, respectively, i.e.,

$$
\mathfrak{p e}_{i}=\left\{X \in \mathfrak{g} l(v) \mid \psi_{i}(X)=-X\right\} \text { for } i=1,2
$$

It is easy to see that the restrictions of $\psi_{1}$ and $\psi_{2}$ onto $\mathfrak{g}_{\overline{0}}$ coincide. Set

$$
\mathfrak{h}^{+}=\left\{x \in \mathfrak{h} \mid \psi_{1}(x)=\psi_{2}(x)=X\right\},
$$

where $\mathfrak{h}$ is Cartan subalgebra in $\mathfrak{g}$.

Let $\lambda$ be a partition and $V^{\lambda}$ the corresponding $\mathfrak{g}$-submodule in the tensor algebra of the identity representation, see Se1.

It follows from [Se4] that, (in Frobenius's notations $\mathrm{Ma}$ )

$$
V^{\lambda} \text { contains a } \mathfrak{p} \mathfrak{e}_{1} \text {-invariant vector if } \lambda=\left(\alpha_{1}, \ldots, \alpha_{p} \mid \alpha_{1}+1, \ldots, \alpha_{p}+1\right)
$$

and

$$
V^{\lambda} \text { contains a } \mathfrak{p e}_{2} \text {-invariant vactor if } \lambda=\left(\alpha_{1}+1, \ldots, \alpha_{p}+1 \mid \alpha_{1}, \ldots, \alpha_{p}\right) \text {. }
$$


Similarly,

$\left(V^{\lambda}\right)^{*}$ contains a $\mathfrak{p} \mathfrak{e}_{1}$-invariant vactor if $\lambda=\left(\alpha_{1}+1, \ldots, \alpha_{p}+1 \mid \alpha_{1}, \ldots, \alpha_{p}\right)$

and

$$
\left(V^{\lambda}\right)^{*} \text { contains a } \mathfrak{p e}_{2} \text {-invariant vactor if } \lambda=\left(\alpha_{1}, \ldots, \alpha_{p} \mid \alpha_{1}+1, \ldots, \alpha_{p}+1\right) \text {. }
$$

Now, let $\lambda=\left(\alpha_{1}+1, \ldots, \alpha_{p}+1 \mid \alpha_{1}, \ldots, \alpha_{p}\right)$, let $v_{\lambda}^{*} \in\left(V^{\lambda}\right)^{*}$ be a $\mathfrak{p} \mathfrak{e}_{1}$-invariant vector, $v_{\lambda} \in\left(V^{\lambda}\right)$ be a $\mathfrak{p e}_{2}$-invariant vector, and let $\varphi_{\lambda}=\Theta\left(v_{\lambda}^{*}, v_{\lambda}\right)$ be the corresponding bispherical function. In $\mathfrak{h}^{+}$, select the basis $\left\{e_{i i}^{+}=\frac{1}{2}\left(e_{i i}+\psi_{1}\left(e_{i i}\right)\right) \mid i \in I_{\overline{0}}\right\}$ and let $\varepsilon_{i}$ be the left dual vectors.

Let $e_{i j}$ be the basis of matrix units in $\mathfrak{g l}(V)$. Set

$$
e_{i j}^{(1)}=e_{i j}, \quad e_{i j}^{(k)}=\sum_{i \in I}(-1)^{p} e_{i p} e_{p j}^{(k-1)}
$$

It is easy to verify that

$$
\left[e_{i j}, e_{p q}^{(k)}\right]=\delta_{j p} e_{i p}^{(k)}-(-1)^{(i+j)(p+q)} \delta_{i q} e_{p j}^{(k)} .
$$

This easily implies that $z_{k}=\sum_{i \in I} e_{i i}^{(k)} \in Z(\mathfrak{g})$.

1.7. Theorem . i) Each two-sided invariant with respect to $\mathfrak{p} \mathfrak{e}_{1}$ and $\mathfrak{p} \mathfrak{e}_{2}$ functional on $U(\mathfrak{g})$ is uniquely determined by its restriction onto $S\left(\mathfrak{h}^{+}\right)$.

ii) Every $z \in Z(\mathfrak{g})$ uniquely determines a differential operator $\Omega_{(z)}$ on the space $S\left(\mathfrak{h}^{+}\right)^{*}$ of restrictions of invariant functionals.

iii) If $z=z_{k}$, then $\Omega_{\left(z_{k}\right)}=\tilde{\Omega}_{k}$.

iv) The functional $\varphi_{\lambda}$ coincides, up to a scalar multiple, with $Q_{\lambda}\left(e^{\varepsilon_{1}}, \ldots, e^{\varepsilon_{k}}\right)$.

\section{§2. The Algebra dual to the Enveloping AlgEBRA}

In this section we follow Dixmier's book [Dix] applied mutatis mutandis to Lie superalgebras.

2.1. Let $\mathfrak{g}$ be a Lie superalgebra. We endow $U(\mathfrak{g})^{*}$ with a coalgebra structure by setting

$$
c: \mathfrak{g} \longrightarrow U(\mathfrak{g}) \otimes U(\mathfrak{g}), \quad c(x)=x \otimes 1+1 \otimes x \text { for any } x \in \mathfrak{g},
$$

so that ${ }^{t} c(x)=c\left({ }^{t} x\right)$ where the first $t$ is the principal antiautomorphism of the superalgebra $U(\mathfrak{g}) \otimes U(\mathfrak{g})=U(\mathfrak{g} \oplus \mathfrak{g})$, see sec. 1.3.

2.2. Lemma . Let $\operatorname{dim} \mathfrak{g}=(n \mid m)$. Then $U(\mathfrak{g})^{*}$ is isomorphic to the supercommutative superalgebra of formal power series in $n$ even and $m$ odd indeterminates.

Proof. Let $\mathfrak{g}_{\overline{0}}=\operatorname{Span}\left(e_{1}, \ldots, e_{n}\right), \mathfrak{g}_{\overline{1}}=\operatorname{Span}\left(e_{\overline{1}}, \ldots, e_{\bar{m}}\right), I_{\overline{0}}=\{1, \ldots, n\}, I_{\overline{1}}=\{\overline{1}, \ldots, \bar{m}\}$.

Denote:

$$
M=\left\{\nu=\left(\nu_{1}, \ldots, \nu_{n}, \nu_{\bar{i}}, \ldots, \nu_{\bar{m}}\right) \mid \nu_{i} \in \mathbb{Z}_{\geq 0} \text { if } i \in I_{\overline{0}} \text { and } \nu_{i} \in\{0,1\} \text { if } i \in I_{\overline{1}}\right\} .
$$

For $\nu \in M$, set

$$
e_{\nu}=\frac{e_{1}^{v_{1}}}{\nu_{1} !} \cdots \frac{e_{n}^{v_{n}}}{\nu_{n} !} \frac{e_{\overline{1}}^{v_{\overline{1}}}}{\left(\nu_{\overline{1}}\right) !} \cdots \frac{e_{\bar{m}}^{v_{\bar{m}}}}{\left(\nu_{\bar{m}}\right) !}
$$

and let $t_{1}, \ldots, t_{n}, t_{\overline{1}}, \ldots, t_{\bar{m}}$ be the set of even and odd supercommuting indeterminates. The correspondence

$$
U(\mathfrak{g})^{*} \ni L \mapsto \sum_{\nu \in M} L\left(e_{\nu}\right) t_{\bar{m}}^{\nu_{\bar{m}}} \ldots t_{\overline{1}}^{\nu_{\overline{1}}} t_{n}^{\nu_{n}} \ldots t_{1}^{\nu_{1}}
$$

determines the homomorphism desired. 
2.3. Left and right coregular representations. For any $u, v \in U(\mathfrak{g})$ and $L \in U(\mathfrak{g})^{*}$ set

$$
\left(L^{*}(u) L\right)(v)=(-1)^{u L} L\left({ }^{t} u v\right) \text { and }\left(R^{*}(u) L\right)(v)=(-1)^{u(L+v)} L(v u) .
$$

The following statements are easy to verify:

i) $u \longrightarrow L^{*}(u)$ is a representation of $U(\mathfrak{g})$ in $U(\mathfrak{g})^{*}$ (we call it the left regular one);

ii) $u \longrightarrow R^{*}(u)$ is a representation of $U(\mathfrak{g})$ in $U(\mathfrak{g})^{*}$ (we call it the right regular one);

iii) If $x \in \mathfrak{g}$, then $L^{*}(x)$ and $R^{*}(x)$ are superdifferentiations of the algebra $U(\mathfrak{g})^{*}$.

Observe also that algebra $U(\mathfrak{g})^{*}$ possesses an automorphism

$$
L \mapsto L^{T}: L^{T}(u)=L\left({ }^{t} u\right) \text { for any } u \in U(\mathfrak{g}), L \in U(\mathfrak{g})^{*}
$$

2.4. Matrix coefficients. Let $V$ be a $\mathfrak{g}$-module, $V^{*}$ the dual module, let $v \in V$ and $v^{*} \in V^{*}$. Let $\pi: \mathfrak{g} \longrightarrow \mathfrak{g l}(V)$ be the corresponding representation. Denote by $\Theta^{\pi}\left(v^{*}, v\right)$ the linear form on $U(\mathfrak{g})^{*}$ :

$$
\Theta^{\pi}\left(v^{*}, v\right)(u)=(-1)^{u v} v^{*}(\pi(u) v) .
$$

Finally, denote by $C(\pi)$ (or $C(V)$ ) the subalgebra in $U(\mathfrak{g})^{*}$ generated by $\Theta^{\pi}\left(v^{*}, v\right)$ for all $v^{*} \in V^{*}$ and $v \in V$.

\subsubsection{Lemma .}

$$
\Theta^{\pi_{1} \otimes \pi_{2}}\left(v_{1}^{*} \otimes v_{2}^{*}, v_{1} \otimes v_{2}\right)=(-1)^{v_{1} v_{2}^{*}} \Theta^{\pi_{1}}\left(v_{1}^{*}, v_{1}\right) \otimes \Theta^{\pi_{2}}\left(v_{2}^{*}, v_{2}\right)
$$

Proof. We have

$$
\begin{aligned}
& \Theta^{\pi_{1} \otimes \pi_{2}}\left(v_{1}^{*} \otimes v_{2}^{*}, v_{1} \otimes v_{2}\right)\left(u_{1} \otimes u_{2}\right) \\
= & (-1)^{\left(u_{1}+u_{2}\right)\left(v_{1}+v_{2}\right)} v_{1}^{*} \otimes v_{2}^{*}\left(\pi_{1}\left(u_{1}\right) \otimes \pi_{2}\left(u_{2}\right)\left(v_{1} \otimes v_{2}\right)\right) \\
= & (-1)^{\left(u_{1}+u_{2}\right)\left(v_{1}+v_{2}\right)+v_{1} u_{2}+v_{2}^{*}\left(u_{1}+v_{1}\right)} v_{1}^{*}\left(\pi_{1}\left(u_{1}\right) v_{1}\right) v_{2}^{*}\left(\pi_{2}\left(u_{2}\right) v_{2}\right) \\
= & \Theta^{\pi_{1}}\left(v_{1}^{*}, v_{1}\right) \otimes \Theta^{\pi_{2}}\left(v_{2}^{*}, v_{2}\right)\left(u_{1} \otimes u_{2}\right) \\
= & (-1)^{u_{1}\left(v_{2}+v_{2}^{*}\right)} \Theta^{\pi_{1}}\left(v_{1}^{*}, v_{1}\right)\left(u_{1}\right) \Theta^{\pi_{2}}\left(v_{2}^{*}, v_{2}\right)\left(u_{2}\right) \\
= & (-1)^{u_{1}\left(v_{2}+v_{2}^{*}\right)+v_{1} u_{1}+v_{2} u_{2}} v_{1}^{*}\left(\pi_{1}\left(u_{1}\right) v_{1}\right) v_{2}^{*}\left(\pi_{2}\left(u_{2}\right) v_{2}\right) .
\end{aligned}
$$

2.4.2. Lemma . The map $V^{*} \otimes V \longrightarrow U(\mathfrak{g})$ given by $v^{*} \otimes v \mapsto \Theta^{\pi}\left(v^{*}, v\right)$ is a $\mathfrak{g} \oplus \mathfrak{g}$-module homomorphism; here we consider $U(\mathfrak{g})^{*}$ as a $\mathfrak{g} \oplus \mathfrak{g}$-module with respect to the left and right coregular representations. If $V$ is irreducible, the above map is an isomorphism.

Proof. Clearly, there exists a linear map $\varphi: V^{*} \otimes V \longrightarrow U(\mathfrak{g})^{*}$ such that $\varphi\left(v^{*} \otimes v\right)=\Theta\left(v^{*}, v\right)$. Let $x \in \mathfrak{g}$. Then

$$
\begin{aligned}
& \varphi\left((x \otimes 1)\left(v^{*} \otimes v\right)\right)(u)=\varphi\left(x v^{*} \otimes v\right)(u)= \\
& \Theta\left(x v^{*}, v\right)(u)=(-1)^{v u}\left(x v^{*}\right)(u)=(-1)^{v u+\overline{1}+x v^{*}} v^{*}(x u) .
\end{aligned}
$$


On the other hand,

$$
\begin{aligned}
L^{*}(x) \varphi\left(v^{*}, v\right)(u) & =(-1)^{x\left(v+v^{*}\right)+\overline{1}} \Theta\left(v^{*}, v\right)(x u) \\
& =(-1)^{x\left(v+v^{*}\right)+\overline{1}+(x+u) v} v^{*}(x u v) \\
& =(-1)^{u v+\overline{1}+x v^{*}} v^{*}(x u)
\end{aligned}
$$

Further,

$\varphi\left((1 \otimes x)\left(v^{*} \otimes v\right)\right)(u)=(-1)^{x v^{*}} \varphi\left(v^{*} \otimes x v\right)(u)=(-1)^{x v^{*}} \Theta\left(v^{*}, x v\right)(u)=(-1)^{x v^{*}+u(x+v)} v^{*}(u x v)$.

On the other hand,

$$
\begin{aligned}
& R^{*}(x) \varphi\left(v^{*} \otimes v\right)(u)=(-1)^{x\left(v^{*}+v+u\right)} \varphi\left(v^{*} \otimes v\right)(u x)=(-1)^{x\left(v^{*}+v+u\right)} \Theta\left(v^{*} \otimes v\right)(u x)= \\
& (-1)^{x\left(v^{*}+v+u\right)+(u+x) v} v^{*}(u x v)=(-1)^{x v^{*}+u(x+v)} v^{*}(u x v) .
\end{aligned}
$$

This proves the first statement. The second one is obvious.

2.4.3. Lemma . Let $\rho$ be the tensor product of representations $\pi_{1}$ and $\pi_{2}$. Then

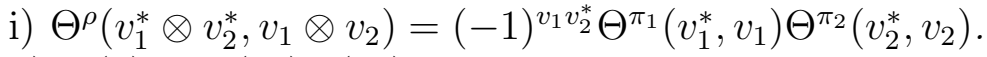

ii) $C(\rho)=C\left(\pi_{1}\right) C\left(\pi_{2}\right)$.

iii) If $\pi$ is finite dimensional, then $\left(\Theta^{\pi}\left(v^{*}, v\right)\right)^{T}=(-1)^{v v^{*}} \Theta^{\pi^{*}}\left(v, v^{*}\right)$.

Proof. i)

$$
\begin{aligned}
(-1)^{v_{1} v_{2}^{*}}\left(\Theta^{\pi_{1}}\left(v_{1}^{*}, v_{1}\right) \Theta^{\pi_{2}}\left(v_{2}^{*}, v_{2}\right)\right)(u) & =(-1)^{v_{1} v_{2}^{*}}\left(\Theta^{\pi_{1}}\left(v_{1}^{*}, v_{1}\right) \otimes \Theta^{\pi_{2}}\left(v_{2}^{*}, v_{2}\right)\right)(C(u)) \\
& =(-1) \Theta^{\pi_{1} \otimes \pi_{2}}\left(v_{1}^{*} \otimes v_{2}^{*}, v_{1} \otimes v_{2}\right)(C(u)) \\
& =(-1)^{\left(v_{1}+v_{2}\right) u}\left(v_{1}^{*} \otimes v_{2}^{*}\right)\left(\pi_{1} \otimes \pi_{2} \cdot C(u)\left(v_{1} \otimes v_{2}\right)\right. \\
& =(-1)^{\left(v_{1}+v_{2}\right) u}\left(v_{1}^{*} \otimes v_{2}^{*}\right)\left(\rho(u)\left(v_{1} \otimes v_{2}\right)\right) \\
& =\Theta^{\rho}\left(v_{1}^{*} \otimes v_{2}^{*}, v_{1} \otimes v_{2}\right)(u) .
\end{aligned}
$$

ii) follows from i)

iii)

$$
\begin{aligned}
\left(\Theta^{\pi}\left(v^{*}, v\right)\right)^{T}(u) & =\Theta^{\pi}\left(v^{*}, v\right)\left({ }^{t} u\right) \\
& =(-1)^{u v} v^{*}\left(\pi\left({ }^{t} u\right)(v)\right) .
\end{aligned}
$$

On the other hand,

$$
\begin{aligned}
\Theta^{\pi^{*}}\left(v, v^{*}\right)(u) & =(-1)^{v^{*} u} v\left(\pi^{*}(u) v^{*}\right) \\
& =(-1)^{v^{*} u+v\left(u+v^{*}\right)}\left(\pi^{*}(u) v^{*}\right)(v) \\
& =(-1)^{v^{*} u+v\left(u+v^{*}\right)+u v^{*}} v^{*}\left(\pi\left({ }^{t} u\right) v\right) .
\end{aligned}
$$


2.4.4. Lemma . Let $V$ be a $\mathfrak{g}$-module. Consider the map

$$
\begin{aligned}
& \varphi: V^{*} \otimes V \otimes V^{*} \otimes V \longrightarrow U(\mathfrak{g})^{*} \\
& \varphi\left(v_{1}^{*} \otimes v_{1} \otimes v_{2}^{*} \otimes v_{2}\right)=(-1)^{v_{1}^{*} v_{2}^{*}+v_{2}^{*} v_{1}+v_{1}^{*} v_{1}}\left(\Theta\left(v_{2}^{*}, v_{1}\right)\right)^{T} \cdot \Theta\left(v_{1}^{*}, v_{2}\right) .
\end{aligned}
$$

If we consider $V^{*} \otimes V \otimes V^{*} \otimes V=\left(V^{*} \otimes V\right) \otimes\left(V^{*} \otimes V\right)$ as a $\mathfrak{g} \oplus \mathfrak{g}$-module in such a way that the first two factors is one $\mathfrak{g}$-module and the last two ones is the other module, then $\varphi$ is a $\mathfrak{g} \oplus \mathfrak{g}$-module homomorphism. (Recall that we consider $U(\mathfrak{g})^{*}$ as a $\mathfrak{g} \oplus \mathfrak{g}$-module with respect to the simultaneous left and right coregular representations.)

Proof. Let $x \in \mathfrak{g}$. Then

$$
\begin{aligned}
& \left.\varphi\left((x \otimes 1)\left(v_{1}^{*} \otimes v_{1} \otimes v_{2}^{*} \otimes v_{2}\right)\right)=\varphi\left(\left(x v_{1}^{*} \otimes v_{1}+(-1)^{x v_{1}^{*}} v_{1}^{*} \otimes x v_{1}\right) \otimes v_{2}^{*} \otimes v_{2}\right)\right) \\
& =(-1)^{v_{2}^{*}\left(x+v_{1}^{*}\right)+v_{2}^{*} v_{1}+\left(x+v_{1}^{*}\right) v_{1}}\left(\Theta\left(v_{2}^{*}, v_{1}\right)\right)^{T} \Theta\left(x v_{1}^{*}, v_{2}\right)+
\end{aligned}
$$

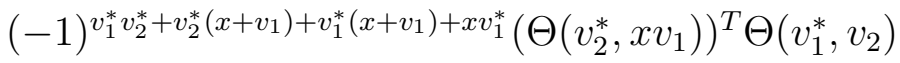

$$
\begin{aligned}
& =(-1)^{v_{1}^{*} v_{2}^{*}+v_{2}^{*} v_{1}+v_{1}^{*} v_{1}} L^{*}(x)\left(\Theta\left(v_{2}^{*}, v_{1}\right)\right)^{T} \Theta\left(v_{1}^{*}, v_{2}\right)+ \\
& (-1)^{v_{1}^{*} v_{2}^{*}+v_{2}^{*} v_{1}+v_{1}^{*} v_{1}+x\left(v_{2}^{*}+v_{1}\right)}\left(\Theta\left(v_{2}^{*}, v_{1}\right)\right)^{T} L^{*}(x) \Theta\left(x v_{1}^{*}, v_{2}\right) \\
& =(-1)^{v_{1}^{*} v_{2}^{*}+v_{2}^{*} v_{1}+v_{1}^{*} v_{1}} L^{*}(x)\left[\left(\Theta\left(v_{2}^{*}, v_{1}\right)\right)^{T} \Theta\left(v_{1}^{*}, v_{2}\right)\right] \\
& =L^{*}(x) \varphi\left(v_{1}^{*} \otimes v_{1} \otimes v_{2}^{*} \otimes v_{2}\right) .
\end{aligned}
$$

The identity

$$
\varphi\left((1 \otimes x)\left(v_{1}^{*} \otimes v_{1} \otimes v_{2}^{*} \otimes v_{2}\right)\right)=R^{*}(x) \varphi\left(v_{1}^{*} \otimes v_{1} \otimes v_{2}^{*} \otimes v_{2}\right)
$$

is similarly verified.

2.4.5. Lemma . Let $V$ be a finite dimensional $\mathfrak{g}$-module, $\left\{v_{i}\right\}_{i \in I}$ its basis, $\left\{v_{i}^{*}\right\}_{i \in I}$ the left dual basis of $V^{*}$. Then

$$
\sum_{i}\left(\Theta\left(v^{*}, v_{i}\right)\right)^{T} \Theta\left(v_{i}^{*}, v\right)=v^{*}(v) \cdot \varepsilon,
$$

where $v \in V, v^{*} \in V^{*}$ and $\varepsilon \in U(\mathfrak{g})^{*}$ is the counit.

Proof. The functional $\varepsilon$ is uniquely, up ot a scalar multiple, characterized by its invariance with respect to the right coregular representation. Further,

$$
\omega=\sum(-1)^{i} v_{i}^{*} \otimes v_{i}
$$

is an invariant of the $\mathfrak{g}$-module $V^{*} \otimes V$. Hence, by Lemma 2.4.4,

$$
\varphi\left(\sum(-1)^{i} v_{i}^{*} \otimes v_{i} \otimes v^{*} \otimes v\right)
$$

is an invariant with respect to the right coregular representation on $U(\mathfrak{g})$, i.e., $\varphi$ is an invariant functional on $U(\mathfrak{g})$. Hence, $\varphi\left(\omega \otimes v^{*} \otimes v\right)=\alpha \varepsilon$.

On the other hand,

$$
\begin{aligned}
& \varphi\left(\omega \otimes v^{*} \otimes v\right)=\sum(-1)^{i)} \varphi\left(v_{i}^{*} \otimes v_{i} \otimes v^{*} \otimes v\right)= \\
& \sum\left(\Theta\left(v^{*}, v_{i}\right)\right)^{T} \cdot \Theta\left(v_{i}^{*}, v\right) .
\end{aligned}
$$


Hence,

$$
\sum_{i}\left(\Theta\left(v^{*}, v_{i}\right)\right)^{T} \cdot \Theta\left(v_{i}^{*}, v\right)=\alpha \varepsilon .
$$

To find $\alpha$, let us substitute $u=1$ into both parts of the identity. We obtain:

$$
\alpha=\alpha \varepsilon(1)=\sum\left(\left(\Theta\left(v^{*}, v_{i}\right)\right)^{T} \cdot \Theta\left(v_{i}^{*}, v\right)(1)=\sum v^{*}\left(v_{i}\right) v_{i}^{*}(v)=v^{*}(v) .\right.
$$

Let $\mathfrak{g}=\mathfrak{g} l(V)$ and let $\mathfrak{A}$ be the subalgebra in $U(\mathfrak{g})^{*}$ generated by $C(V)$ and $C\left(V^{*}\right)$. It is not difficult to verify that $\mathfrak{A}$ is invariant with respect to the left and right coregular representations.

$\S 3$. Bispherical Functions and Radial parts of LAplace operators For the TRIPLE $(\mathfrak{q}(n), \mathfrak{q}(n) \oplus \mathfrak{q}(n), \mathfrak{q}(n))$

3.1. Let $\mathfrak{g}=\mathfrak{q}(n)$, let $Z(\mathfrak{g})$ be the center of $U(\mathfrak{g})$ and $\mathfrak{h}=\operatorname{Span}\left(e_{i i}, f_{j j} \mid i, j \in I_{\overline{0}}\right)$ the Cartan subalgebra (for definition of $e_{i j}$ and $f_{i j}$ see sec. 1.4.2). Let $\mu \in \mathfrak{h}_{0}^{*}$ and $M(\mu)$ the coresponding Verma module with the highest weight with respect to the decomposition $\mathfrak{g}=\mathfrak{n}_{-} \oplus \mathfrak{h} \oplus \mathfrak{n}_{+}$, where $\mathfrak{n}_{-}=\operatorname{Span}\left(e_{i j}, f_{i j} \mid i>j\right)$ and $\left.\mathfrak{n}_{+}=\operatorname{Span}\left(e_{i j}, f_{i j}\right) \mid i<j\right)$, see [Se4], [P]]. Such module $M(\mu)$ has a central character, i.e., there exists a homomorphism

$$
\chi_{\mu}: Z(\mathfrak{g}) \longrightarrow \mathbb{C}, \quad z v=\chi_{\mu}(z) v \text { for any } v \in M(\mu) \text { and } z \in Z(\mathfrak{g}) .
$$

Let $\varepsilon_{i}$ be the left dual vector to $e_{i i}$. Set $R^{+}=\left\{\varepsilon_{i}-\varepsilon_{j} \mid i<j\right\}$ and

$$
B(\mu)=\left\{\nu \mid \nu=\lambda-\sum n_{\alpha} \alpha \text { for any } n_{\alpha} \in \mathbb{Z}_{\geq 0}, \text { where } \alpha \in R^{+} \text {, and } \chi_{\nu}=\chi_{\mu}\right\} \text {. }
$$

3.2. Lemma . Let $L(\mu)$ be an irreducible module with highest weight $\mu$. Then

$$
\operatorname{ch} L(\mu)=\sum_{\nu \in B(\mu)} C_{\mu \nu} \operatorname{ch} M(\nu)
$$

where for any fixed $\tau \in \mathfrak{h}_{\overline{0}}^{*}$ only a finite number of summands in the right hand side contains $\tau$ as a weight.

Proof. Let $\alpha_{1}, \ldots, \alpha_{n-1}$ be a base (system of simple roots) in the root system $R$. Set

$$
h t(\nu)=\sum_{1}^{n-1} k_{i} \quad \text { for any } \nu=\mu-\sum_{i=1}^{n-1} k_{i} \alpha_{i} .
$$

By induction on $t$ we prove that

$$
\operatorname{ch} L(\mu)=\sum_{\nu \in B(\mu), h t(\nu) \leq t} C_{\mu \nu} \operatorname{ch} M(\nu)+\sum_{i=1}^{m(t)} \operatorname{ch} V_{i}
$$

for any $\tau \in \operatorname{Supp} \operatorname{ch} V_{i}, h t(\tau)>t, \chi_{V_{i}}=\chi_{\mu}$ and $\operatorname{Supp} \operatorname{ch} V_{i} \subset B(\mu)$.

Let $t=0$. Then we obtain an exact sequence

$$
0 \longrightarrow N \longrightarrow M(\mu) \longrightarrow L(\mu) \longrightarrow 0
$$

with $N \subset \underset{\nu \neq \mu}{\oplus} M(\mu)_{\nu}$. Therefore, $h t(\nu)>0$ and $\operatorname{ch} L(\mu)=\operatorname{ch} M(\mu)-\operatorname{ch}(N)$; moreover, Supp $\operatorname{ch} N \subset B(\mu)$.

Let (3.2.2) hold. Consider the set $h t(\nu)$, where $\nu \in \operatorname{Supp} \operatorname{ch} V_{i}$ and let $k$ be the least element of this set. Clearly, $k>t$. There exists then a finite number of vectors $v_{1}^{i}, \ldots, v_{l}^{i} \in V_{i}$ with weights $\mu_{1 i}, \ldots, \mu_{l i}$ such that $h t\left(\mu_{1 i}\right)=h t\left(\mu_{2 i}\right) \cdots=k$. For all the other weights $\nu$ of $V_{i}$ we 
have $h t(\nu)>k \geq t+1$. The vectors $v_{1}^{i}, \ldots, v_{l}^{i}$ are, obviously, the highest weight ones, so we have an exact sequence

$$
0 \longrightarrow N \longrightarrow \bigoplus_{j=1}^{l} M\left(\mu_{j i}\right) \longrightarrow V_{i} \longrightarrow K \longrightarrow 0 .
$$

This implies:

$$
\operatorname{ch} V_{i}=\sum_{j=1}^{l} \operatorname{ch} M\left(\mu_{j i}\right)+\operatorname{ch} K-\operatorname{ch} N
$$

and if $\nu \in \operatorname{Supp} \operatorname{ch} K \cup \operatorname{Supp} \operatorname{ch} N$, then $h t(\nu)>t+1$. This proves (3.2.2).

Let $\nu \in \mathfrak{h}_{\overline{0}}^{*}$ be any weight. Set $t=h t(\nu)$; apply (3.2.2) to see that $\nu \notin \cup \operatorname{Supp} \operatorname{ch} V_{i}$. This proves (3.2.1).

3.3. Lemma . Let $z_{3}=\sum e_{i i}^{(3)}$ (see 1.4.2) and let $v_{\mu}$ be the highest weight vector of the $\mathfrak{g}$-module $M(\mu)$. Then

$$
z_{3} v_{\mu}=\left(\sum \mu_{i}^{3}-\left(\sum \mu_{i}\right)^{2}\right) v_{\mu}
$$

Proof. First, observe that

$$
\begin{aligned}
& e_{i i}^{(3)}=\sum_{j=1}^{n} e_{i j} e_{j i}^{(2)}+\sum_{j=1}^{n} f_{i j} f_{j i}^{(2)}=\sum_{i<j} e_{i j} e_{j i}^{(2)}+\sum_{i<j} f_{i j} f_{j i}^{(2)} \\
& +\sum_{i>j} e_{i j} e_{j i}^{(2)}+\sum_{i>j} f_{i j} f_{j i}^{(2)}+\sum e_{i i} e_{i i}^{(2)}+\sum f_{i i} f_{i i}^{(2)}
\end{aligned}
$$

But, as is easy to verify, $e_{i j}^{(2)} v_{\mu}=f_{i j}^{(2)} v_{\mu}=0$. Therefore,

$$
\begin{aligned}
& z_{3} v_{\mu}=\left(\sum e_{i i} e_{i i}^{(2)}+\sum f_{i i} f_{i i}^{(2)}\right) v_{\mu}+\left(\sum_{i<j} e_{i j} e_{j i}^{(2)}+\sum f_{i j} f_{j i}^{(2)}\right) v_{\mu} \\
& =\left(\sum e_{i i} e_{i i}^{(2)}+\sum f_{i i} f_{i i}^{(2)}+\sum_{i<j} e_{i i}^{(2)}-e_{j j}^{(2)}+\sum_{i<j} e_{j j}^{(2)}-e_{i i}^{(2)}\right) v_{\mu} \\
& =\left(\sum e_{i i} e_{i i}^{(2)}+\sum f_{i i} f_{i i}^{(2)}\right) v_{\mu} .
\end{aligned}
$$

Further, it is easy to verify that

$$
e_{i i}^{(2)} v_{\mu}=\left(\mu_{i}^{2}-\mu_{i}-2 \sum_{k>i} \mu_{k}\right) v_{\mu} \text { and } f_{i i}^{(2)} v_{\mu}=0 .
$$

Hence, $z_{3} v_{\mu}=\left(\sum \mu_{i}^{3}-\left(\sum \mu_{i}\right)^{2}\right) v_{\mu}$.

3.4. Corollary . Let $\delta=\prod_{\alpha \in R^{+}} \frac{e^{\alpha / 2}+e^{-\alpha / 2}}{e^{\alpha / 2}-e^{-\alpha / 2}}$ (see 1.4.2) and let $\Omega_{3}^{*}=\sum \partial_{i}^{3}-\left(\sum \partial_{i}\right)^{2}$, where $\partial_{i} e^{l}=l\left(e_{i}\right) e^{l}$. Then $\Omega_{3}^{*} \delta^{-1}=0$ and $\left(\sum \partial_{i}^{3}\right) \delta^{-1}=0$.

Proof. Let us prove a more general statement; namely, let $\varphi_{\mu}$ be the character of an irreducible $\mathfrak{g}$-module with highest weight $\mu$. Then

$$
\delta^{-1} \varphi_{\mu} \text { is an eigenfunction of } \Omega_{3}^{*} \text { with eigenvalue } \sum \mu_{i}^{3}-\left(\sum \mu_{i}\right)^{3} \text {. }
$$

Indeed, by Lemma 3.2

$$
\varphi_{\mu}=\sum_{\nu \in B(\mu)} C_{\mu \nu} \operatorname{ch} M(\nu)
$$


By multiplying both parts of the inequality by $\delta^{-1}$ we obtain

$$
\delta^{-1} \varphi_{\mu}=\sum_{\nu \in B(\mu)} \tilde{C}_{\mu \nu} e^{\nu}
$$

If $z \in Z(\mathfrak{g})$, then $z v_{\mu}=\chi_{\mu}(z) v_{\mu}$. Since $\nu \in B(\mu)$, it follows that $\chi_{\mu}(z)=\chi_{\nu}(z)$. But by Lemma 3.3

$$
\chi_{\mu}\left(z_{3}\right)=\sum \mu_{i}^{3}-\left(\sum \mu_{i}\right)^{2}=\chi_{\nu}\left(z_{3}\right)=\sum \nu_{i}^{3}-\left(\sum \nu_{i}\right)^{2} .
$$

So for $\Omega_{3}^{*}=\sum \partial_{i}^{3}-\left(\sum \partial_{i}\right)^{2}$, we have

$$
\begin{aligned}
& \Omega_{3}^{*} \delta^{-1} \varphi_{\mu}=\sum_{\nu \in B(\mu)} \Omega_{3}^{*}\left(\tilde{C}_{\mu \nu} e^{\nu}\right)=\sum_{\nu \in B(\mu)} \tilde{C}_{\mu \nu}\left(\sum v_{i}^{3}-\left(\sum v_{i}\right)^{2}\right) e^{\nu} \\
& =\left(\sum \mu_{i}^{3}-\left(\sum \mu_{i}\right)^{2}\right) \delta^{-1} \varphi_{\mu} .
\end{aligned}
$$

In particular, applying this statement to the trivial module we obtain $\Omega_{3}^{*} \delta^{-1}=0$, implying $\left(\sum \partial_{i}^{3}\right) * \delta^{-1}=0$.

3.5. Proof of heading i) of Lemma 1.2.3. Let $R=\left\{\varepsilon_{i}-\varepsilon_{j} \mid i \neq j\right\}$ and $R^{+}=\left\{\varepsilon_{i}-\varepsilon_{j} \mid\right.$ $i<j\}$. Set $\Delta_{\alpha}^{+}=e^{\frac{1}{2} \alpha}+e^{-\frac{1}{2} \alpha}$ and $\Delta_{\alpha}^{-}=e^{\frac{1}{2} \alpha}-e^{-\frac{1}{2} \alpha}$ for any $\alpha \in R$. Then

$$
\partial_{i}\left(\Delta_{\alpha}^{+}\right)=\frac{1}{2} \alpha\left(e_{i}\right) \Delta_{\alpha}^{-}, \quad \partial_{i}\left(\Delta_{\alpha}^{-}\right)=\frac{1}{2} \alpha\left(e_{i}\right) \Delta_{\alpha}^{+}
$$

We also set

$$
\varphi_{i}=\sum_{\alpha \in R^{+}} \frac{\alpha\left(e_{i}\right)}{\Delta_{\alpha}^{+} \Delta_{\alpha}^{-}}, \quad \psi_{i}=\sum_{\alpha \in R^{+}} \frac{\alpha^{2}\left(e_{i}\right)}{\left(\Delta_{\alpha}^{+}\right)^{2}}, \quad \Theta_{i}=\sum_{\{\alpha, \beta\} \subset R^{+}} \frac{\alpha\left(e_{i}\right) \beta\left(e_{i}\right)}{\Delta_{\alpha}^{+} \Delta_{\alpha}^{-} \Delta_{\beta}^{+} \Delta_{\beta}^{-}}
$$

where the last sum runs over the two-element subsets of $R^{+}$. It is not difficult to verify that one can express the operator $\hat{\Omega}_{3}=\Omega_{3}+\left(\sum \partial_{i}\right)^{2}$ in the form

$$
\hat{\Omega}_{3}=\sum \partial_{i}^{3}+6 \sum \varphi_{i} \partial_{i}^{2}-6 \sum \psi_{i} \partial_{i}+24 \sum \Theta_{i} \partial_{i} .
$$

It is easy to verify that $\delta^{-1} \partial_{i} \delta=\partial_{i}-2 \varphi_{i}$; hence,

$$
\begin{gathered}
\delta^{-1} \partial_{i}^{2} \delta=\partial_{i}^{2}-4 \varphi_{i} \partial_{i}+4 \varphi_{i}^{2}-2 \partial_{i}\left(\varphi_{i}\right) \\
\delta^{-1} \partial_{i}^{3} \delta=\partial_{i}^{3}-6 \varphi_{i} \partial_{i}^{2}+3\left(4 \varphi_{i}^{2}-2 \partial_{i}\left(\varphi_{i}\right)\right) \partial_{i}-8 \varphi_{i}^{3}-2 \partial_{i}^{2}\left(\varphi_{i}\right)+12 \varphi_{i} \partial_{i}\left(\varphi_{i}\right)
\end{gathered}
$$

Therefore,

$$
\begin{aligned}
& \delta^{-1} \hat{\Omega} \delta=\sum_{i}\left[\partial_{i}^{3}+\left(24 \Theta_{i}-6 \psi_{i}-12 \varphi_{i}^{2}-6 \partial_{i}\left(\varphi_{i}\right)\right) \partial_{i}\right. \\
& \left.+\left(16 \varphi_{i}^{3}-2 \partial_{i}^{2}\left(\varphi_{i}\right)-48 \Theta_{i} \varphi_{i}+12 \psi_{i} \varphi_{i}\right)\right] .
\end{aligned}
$$

Direct calculations show that

$$
24 \Theta_{i}-6 \psi_{i}-12 \varphi_{i}^{2}-6 \partial_{i}\left(\varphi_{i}\right)=0 .
$$

Hence,

$$
\delta^{-1} \hat{\Omega} \delta=\sum \partial_{i}^{3}+f
$$

But, $\hat{\Omega}(1)=0$, so

$$
\left(\delta^{-1} \hat{\Omega} \delta\right) \cdot\left(\delta^{-1}\right)=\left(\sum \partial_{i}^{3}\right)\left(\delta^{-1}\right)+f \delta^{-1}=0 .
$$

But due to Corollary 3.4, $\left(\sum \partial_{i}^{3}\right)\left(\delta^{-1}\right)=0$; hence, $f \delta^{-1}=0$ and $f=0$. 
3.6. Lemma . Let $\mathfrak{g}, \mathfrak{g}_{1}, \mathfrak{g}_{2}$ be selected as in sec. 1.4 , let I be the left ideal in $U(\mathfrak{g} \oplus \mathfrak{g})$ generated by $\mathfrak{g}_{2}$ and $M=U(\mathfrak{g} \oplus \mathfrak{g}) / I$. Let $\sigma: \mathfrak{g} \longrightarrow \mathfrak{g} \oplus \mathfrak{g}$ be the embedding into the first summand, i.e., $\sigma(x)=(x, 0)$. Let $\tilde{\sigma}: U(\mathfrak{g}) \longrightarrow M$ be the map induced by the homomorphism $U(\mathfrak{g}) \longrightarrow U(\mathfrak{g} \oplus \mathfrak{g})$ that extends $\sigma$ and $\rho(x)=\left(x,(-1)^{x} x\right)$ an isomorphism of $\mathfrak{g}$ with $\mathfrak{g}_{1}$. Then $\tilde{\sigma}(x * u)=\rho(x) \tilde{\sigma}(u)$.

Proof.

$$
\begin{aligned}
\tilde{\sigma}(x * u) \quad & \tilde{\sigma}\left(x u-(-1)^{x(u+\overline{1})} u x\right)=x u \otimes 1-(-1)^{x(u+\overline{1})} u x \otimes 1 \\
= & \quad x u \otimes 1-(-1)^{x(u+\overline{1})} u x \otimes 1-\rho(x) \tilde{\sigma}(u)+\rho(x) \tilde{\sigma}(u) \\
= & \rho(x) \tilde{\sigma}(u)+x u \otimes 1-(-1)^{x(u+\overline{1})} u x \otimes 1 \\
& -\left(x \otimes 1+(-1)^{x} 1 \otimes x\right)(u \otimes 1) \\
= & \rho(x) \tilde{\sigma}(u)-(-1)^{x(u+\overline{1})}(u x \otimes 1+u \otimes x) \\
=\quad & \rho(x) \tilde{\sigma}(u)-(-1)^{x(u+\overline{1})}(u \otimes 1)(x \otimes 1+1 \otimes x) \\
\equiv(\bmod I) & \rho(x) \tilde{\sigma}(u) .
\end{aligned}
$$

3.6.1. Statement of Lemma 1.4 is true. This is a direct corollary of Lemma 3.6.

3.7. Proof of heading i) of Theorem 1.5. Due to [Se4] and [G] we have an isomorphism of $\mathfrak{g}$-modules with respect to the action (1.4.1):

$$
U(\mathfrak{g}) \simeq \operatorname{Ind}_{\mathfrak{g}_{\overline{0}}}^{\mathfrak{g}}\left(U\left(\mathfrak{g}_{\overline{0}}\right)\right)
$$

Therefore, there exists a bijection between the space of $\mathfrak{g}$-invariant with respect to the action (1.4.1) functionals on $U(\mathfrak{g})$ and the space of $\mathfrak{g}_{\overline{0}}$-invariant functionals on $U\left(\mathfrak{g}_{\overline{0}}\right)$. Moreover, any $\mathfrak{g}$-invariant functional is uniquely determined by its restriction onto $U\left(\mathfrak{g}_{\overline{0}}\right)$. On the other hand, every $\mathfrak{g}_{\overline{0}}$-invariant functional is uniquely determined by its restriction onto $U\left(\mathfrak{h}_{\overline{0}}\right)=$ $S\left(\mathfrak{h}_{\overline{0}}\right)$.

3.8. Let $l \in\left(U(\mathfrak{g})^{*}\right)^{\mathfrak{g}}$ and $\varphi_{l}$ the generating function of its restriction onto $S\left(\mathfrak{h}_{\overline{0}}\right)$, i.e.,

$$
\varphi_{l}\left(t_{1}, \ldots, t_{n}\right)=\sum \frac{l\left(e_{11}^{\nu_{1}} \ldots e_{n n}^{\nu_{n}}\right)}{\left(\nu_{1}\right) ! \ldots\left(\nu_{n}\right) !} t_{1}^{\nu_{1}} \ldots t_{n}^{\nu_{n}} .
$$

On $S\left(\mathfrak{h}_{\overline{0}}\right)^{*}$, define the following operators by setting for any $f \in S\left(\mathfrak{h}_{\overline{0}}\right)$ :

$$
\begin{array}{cl}
\left(\partial_{i}^{(k)} l\right)(f)=l\left(e_{i i}^{(k)} f\right), & \left(\delta_{i}^{(k)} l\right)(f)=L\left(f_{i i}^{(k)} f\right), \\
\left(D_{i j}^{(k)} l\right)(f)=l\left(e_{i j} e_{j i}^{(k)} f\right), & \left(\Delta_{i j}^{(k)} l\right)(f)=L\left(f_{i j} f_{j i}^{(k)} f\right) .
\end{array}
$$

3.8.1. Lemma . Let $\alpha=\varepsilon_{i}-\varepsilon_{j}$. Then
i) $D_{i j}^{(k)}=\frac{e^{\alpha}}{e^{\alpha}-1}\left(\partial_{i}^{(k)}-\partial_{j}^{(k)}\right)$.
ii) $\Delta_{i j}^{(k)}=\frac{e^{\alpha}}{e^{\alpha}+1}\left(\partial_{j}^{(k)}+(-1)^{k+1} \partial_{i}^{(k)}\right)$.
iii) $l\left(f \cdot f_{i i} f_{i i}^{(k)}\right)=l\left(f e_{i i}^{(k)}\right)$ for $k$ odd.
iv) $l\left(f \cdot f_{i i} f_{i i}^{(k)}\right)=0$ for $k$ even. 
Proof. i) and ii) are similarly proved. Consider i):

$$
\begin{aligned}
& \left(D_{i j}^{(k)} l\right)(f)=l\left(f e_{i j} e_{j i}^{(k)}\right)=L\left(e_{i j} f(h+\alpha(h)) e_{j i}^{(k)}=l\left(f(h+\alpha(h)) e_{j i}^{(k)} e_{i j}\right)\right. \\
& =l\left(f(h+\alpha(h)) e_{i j} e_{j i}^{(k)}\right)-l\left(f(h+\alpha(h))\left[e_{i j}, e_{j i}^{(k)}\right]\right) \\
& =\left(e^{\alpha} D_{i j}^{(k)} l\right)(f)-l\left(f(h+\alpha(h))\left(e_{i i}^{(k)}-e_{j j}^{(k)}\right)\right) \\
& =\left(e^{\alpha} D_{i j}-e^{\alpha}\left(\partial_{i}^{(k)}-\partial_{j}^{(k)}\right)\right)(l)(f) .
\end{aligned}
$$

This proves i).

iii) We have

$$
\begin{aligned}
& f_{i i} *\left(f \cdot f_{i i}^{(k)}\right)=f_{i i} f \cdot f_{i i}^{(k)}-f \cdot f_{i i}^{(k)} f_{i i} \\
& =f\left(f_{i i} f_{i i}^{(k)}-f_{i i}^{(k)} f_{i i}\right)=f\left(2 f_{i i} f_{i i}^{(k)}-\left[f_{i i}, f_{i i}^{(k)}\right]\right)=2 f f_{i i} \cdot f_{i i}^{(k)}-2 e_{i i}^{(k)} f
\end{aligned}
$$

or $f \cdot f_{i i} \cdot f_{i i}^{(k)}=\frac{1}{2} f_{i i} *\left(f \cdot f_{i i}^{(k)}\right)+f \cdot e_{i i}^{(k)}$. This proves iii). Heading iv $)$ is similar.

\subsubsection{Corollary . Heading iii) of Theorem 1.5 is true.}

3.9. Proof of heading iv) of Theorem 1.5. Let $V^{\lambda}=\operatorname{Span}\left(v_{p}\right)$, where the $v_{p}$ form a basis of $V^{\lambda}$, let the $v_{p}^{*}$ form the left dual basis; set $\omega_{\lambda}^{*}=\sum v_{p}^{*} \otimes v_{p}$, and $\omega_{\lambda}=\sum v_{p} \otimes v_{p}^{*}$.

If $\mathfrak{g}$ is embedded into $\mathfrak{g} \oplus \mathfrak{g}$ as the first summand, and $u \in U(\mathfrak{g})$, then

$$
\begin{aligned}
& \varphi_{\lambda}(u \otimes 1)=\Theta\left(\omega_{\lambda}^{*}, \omega_{\lambda}\right)(u \otimes 1)=\omega_{\lambda}^{*}\left(u \otimes 1\left(\omega_{\lambda}\right)\right) \\
& =\omega_{\lambda}^{*}\left(\sum u v_{p} \otimes v_{p}^{*}\right)=\sum v_{p}^{*}\left(u v_{p}\right)=\operatorname{tr}(u) .
\end{aligned}
$$

In other words, the matrix coefficient $\Theta^{\pi}\left(\omega_{\lambda}^{*}, \omega_{\lambda}\right)$ coincides with the functional $\operatorname{tr}_{V^{\lambda}}(u)$ after restriction onto $U(\mathfrak{g})$. But due to [Se1] we have, up to a scalar multiple,

$$
\left.\operatorname{tr}_{V^{\lambda}}\right|_{\mathfrak{h}_{\overline{0}}}=Q_{\lambda}\left(e^{\varepsilon_{1}}, \ldots, e^{\varepsilon_{n}}\right) \text {. }
$$

3.10. Proof of headings ii) and iii) of Lemma 1.2.3. Heading ii) immediately follows from our proof of Corollary 3.4. Let us prove iii). It is not difficult to see that the algebra $\Omega$ generated by all the $\Omega_{k}, k=1,3,5, \ldots$, is the image of $Z(\mathfrak{q}(n))$ under the homomorphism

$$
r: Z(\mathfrak{q}(n)) \longrightarrow \Omega, \quad r(z) Q_{\lambda}=\varphi(z)(\lambda) Q_{\lambda},
$$

where $\varphi$ is the Harish-Chandra homomorphism, see [Se3]. Therefore, statement of heading iii) can be reformulated as follows:

Let $R_{n}$ be the algebra of polynomials $r\left(t_{1}, \ldots, t_{n}\right)$ symmetric with respect to $\left(t_{1}, \ldots, t_{n}\right)$ and which do not depend on $s$ after substitution $t_{i}=s, t_{j}=-s$. Define an $R_{n}$-action on the algebra generated by $Q_{\lambda}$ by setting

$$
r * Q_{\lambda}=r(\lambda) Q_{\lambda}
$$

If $P$ is an eigenvector for $R_{n}$ and belongs to the subalgebra generated by $Q_{\lambda}$, then $P$ coincides, up to a scalar multiple, with one of the $Q_{\lambda}$.

Indeed, let $P=\sum_{\lambda \in \Lambda} C_{\lambda} Q_{\lambda}$, where $\Lambda$ is the set of partitions of length $\leq n$. Since $r * P=C(r) P$ it follows that $r(\lambda)=$ const for any $\lambda \in \Lambda$. Let $\lambda, \mu \in \Lambda$ and $\lambda \neq \mu$; then 
$r(\lambda)=r(\mu)$ for any $r \in R_{n}$. Hence,

$$
\prod \frac{t-\lambda_{i}}{t+\lambda_{i}}=\prod \frac{t-\mu_{j}}{t+\mu_{j}}
$$

We may assume that $\lambda_{i}>0$ and $\mu_{i}>0$ for all $i$ and $j$. Then the identity

$$
\prod\left(t-\lambda_{i}\right) \prod\left(t+\mu_{j}\right)=\prod\left(t+\lambda_{i}\right) \prod\left(t+\mu_{j}\right)
$$

implies $\prod\left(t-\lambda_{i}\right)=\prod\left(t-\mu_{j}\right)$, hence, $\lambda=\mu$. Contradiction.

$\S 4$. Bispherical Functions and the RAdial parts of LAPlace operators For THE TRIPLE $(\mathfrak{p} \mathfrak{e}(n), \mathfrak{g l}(n \mid n), \mathfrak{p} \mathfrak{e}(n))$

4.1. Let $V$ be an $(n \mid n)$-dimensional superspace, $I=I_{\overline{0}} \cup I_{\overline{1}}=\{1, \ldots, n\} \cup\{\overline{1}, \ldots, \bar{n}\}$, the union of the even and odd indices, see sec. 1.4.1. Let $\left\{e_{i}\right\}_{i \in I}$ be the basis of $V$ consisting of vectors whose parity is equal to that of their indices and $\left\{e_{i j}\right\}_{i, j \in I}$ be the basis fo $\operatorname{Mat}(V)$ consisting of matrix units, cf. sec. 1.4.2.

The supertransposition antiautomorphism in $\mathfrak{g l}(V)$ is in these terms of the form

$$
e_{i j}^{t}=(-1)^{p(i)(p(j)+\overline{1})} e_{j i} .
$$

Define two antiautomorphisms $\psi_{1}$ and $\psi_{2}$ by setting

$$
\psi_{1}(x)=(-1)^{x} \Pi\left(x^{t}\right) \Pi \text { and } \psi_{2}=P \psi_{1} P .
$$

Direct calculations show that

$$
\psi_{1}\left(e_{i j}\right)=(-1)^{p(j)(p(i)+\overline{1})} e_{\bar{j} i}, \quad \psi_{2}\left(e_{i j}\right)=(-1)^{p(i)(p(j)+\overline{1})} e_{\bar{\jmath} \bar{i}} .
$$

Define two Lie subsuperalgebras of $\mathfrak{g l}(V)$ :

$$
\mathfrak{p e}_{1}(V)=\left\{x \in \mathfrak{g l}(V) \mid \psi_{1}(x)=-x\right\}
$$

and

$$
\mathfrak{p e}_{2}(V)=\left\{x \in \mathfrak{g l}(V) \mid \psi_{2}(x)=-x\right\} .
$$

Observe that $\psi_{1}(x)=\psi_{2}(x)$ if $p(x)=0$ and $\psi_{1}(x)=-\psi_{2}(x)$ if $p(x)=1$. Therefore,

$$
\mathfrak{p e}_{1}(V)=\mathfrak{g l}(V)_{\overline{0}}^{-} \oplus \mathfrak{g l}(V)_{\overline{1}}^{-}, \quad \mathfrak{p e}_{2}(V)=\mathfrak{g l}(V)_{\overline{0}}^{-} \oplus \mathfrak{g l}(V)_{\overline{1}}^{+},
$$

where

$$
\begin{aligned}
& \mathfrak{g l}(V)_{\overline{0}}^{-}=\left\{x \in \mathfrak{g l}(V)_{\overline{0}} \mid \psi_{2}(x)=-x\right\}, \\
& \mathfrak{g l}(V)_{\overline{1}}^{-}=\left\{x \in \mathfrak{g l}(V)_{\overline{1}} \mid \psi_{1}(x)=-x\right\}, \\
& \mathfrak{g l}(V)_{\overline{1}}^{+}=\left\{x \in \mathfrak{g l}(V)_{\overline{1}} \mid \psi_{1}(x)=x\right\} .
\end{aligned}
$$

For every $x \in \mathfrak{g l}(V)$, set

$$
x^{+}=\frac{1}{2}\left(x+\psi_{1}(x)\right), \quad x^{-}=\frac{1}{2}\left(x-\psi_{1}(x)\right) .
$$

Also set

$$
\mathfrak{h}^{+}=\operatorname{Span}\left(e_{i i}^{+} \mid i \in I_{\overline{0}}\right) .
$$

4.2. Lemma . For $f \in S\left(\mathfrak{h}^{+}\right)$and $\alpha=\varepsilon_{i}-\varepsilon_{j}$ set

$$
\begin{aligned}
& R_{i j}^{-} f=\frac{1}{2}[f(h-\alpha(h))-f(h+\alpha(h))], \\
& R_{i j}^{+} f=\frac{1}{2}[f(h-\alpha(h))+f(h+\alpha(h))] .
\end{aligned}
$$

Then the following identities hold:

i) $e_{i j}^{-} f=R_{i j}^{+} f e_{i j}^{-}+R_{i j}^{-} f \cdot e_{i j}^{+}$. 
ii) $R_{i j}^{-} f e_{i j} e_{j i}^{(k)}-\left(R_{i j}^{-}-R_{i j}^{+}\right) f \cdot\left[e_{i j}^{-}, e_{j i}^{(k)}\right] \in \mathfrak{p} \mathfrak{e}_{1} U(\mathfrak{g})+U(\mathfrak{g}) \mathfrak{p e}_{2}$ if $p(i)+p(j)=0$.

iii) $R_{i j}^{+} f e_{i j} e_{j i}^{(k)}-\left(R_{i j}^{+}-R_{i j}^{-}\right) f \cdot\left[e_{i j}^{+}, e_{j i}^{(k)}\right] \in \mathfrak{p} \mathfrak{e}_{1} U(\mathfrak{g})+U(\mathfrak{g}) \mathfrak{p e}_{2}$ if $p(i)+p(j)=1$.

iv) $\left[e_{i j}^{-}, e_{j i}^{(k)}\right]=\frac{1}{2}\left[e_{i i}^{(k)}-e_{j j}^{(k)}\right]$ if $p(i)+p(j)=0$.

v) $\left[e_{i j}^{+}, e_{j i}^{(k)}\right]=\frac{1}{2}\left[e_{i i}^{(k)}+e_{j j}^{(k)}\right]$ if $\bar{i} \neq j$ and $p(i)+p(j)=1$.

vi) $\left[e_{i \bar{i}}^{+}, e_{i i}^{(k)}\right]=e_{i i}^{(k)}+e_{\overline{i i}}^{(k)}$ if $p(i)=1$.

vii) $\left[e_{i \bar{i}}^{+}, e_{\bar{i} i}^{(k)}\right]=0$ if $p(i)=0$.

Proof is reduced to a direct verification.

4.3. Proof of heading $\mathbf{i}$ ) of Theorem 1.7. It suffices to prove that $U(\mathfrak{g})=S\left(\mathfrak{h}^{+}\right)+$ $\mathfrak{p} \mathfrak{e}_{1} U(\mathfrak{g})+U(\mathfrak{g}) \mathfrak{p} \mathfrak{e}_{2}$.

Indeed, any element of $U(\mathfrak{g})$ can be represented in the form

$$
u=u_{0}+u_{1} u_{0}^{\prime} u_{2} \text {, where } u_{1} \in U\left(\mathfrak{g}_{\overline{1}}^{-}\right), u_{2} \in U\left(\mathfrak{g}_{\overline{1}}^{+}\right), u_{0}, u_{0}^{\prime} \in U\left(\mathfrak{g}_{\overline{0}}\right) .
$$

Therefore, we may assume that

$$
u=f e_{\alpha_{1}}^{+} \ldots e_{\alpha_{k}}^{+},
$$

where $f \in S\left(\mathfrak{h}^{+}\right)$, and $e_{\alpha_{1}}^{+}, \ldots, e_{\alpha_{k}}^{+} \in \mathfrak{g}_{0}$ are the weight vectors.

Now, induction on $k$. If $k=1$, then $R_{i j}^{-} f e_{i j}^{+}=e_{i j}^{-} f-R_{i j}^{+} f e_{i j}^{-}$by Lemma 4.2.i). Hence, $f e_{i j}^{+} \in \mathfrak{g}_{\overline{0}}^{-} U\left(\mathfrak{g}_{\overline{0}}\right) \mathfrak{g}_{\overline{0}}^{-}$. Let $k>1$. Then

$$
\begin{aligned}
& R_{\alpha_{1}}^{-} f e_{\alpha_{1}}^{+} \ldots e_{\alpha_{k}}^{+} \quad=\quad e_{\alpha_{1}}^{-} f e_{\alpha_{2}}^{+} \ldots e_{\alpha_{n}}^{+}-R_{\alpha_{1}}^{+} f e_{\alpha_{1}}^{-} e_{\alpha_{2}}^{+} \ldots \\
& \equiv{ }_{\left(\bmod \mathfrak{g}_{\overline{0}}^{-} U(\mathfrak{g}) \mathfrak{g}_{\overline{0}}^{-}\right)} \quad-R_{\alpha_{1}}^{+} f e_{\alpha_{1}}^{-} e_{\alpha_{2}}^{+} \ldots e_{\alpha_{n}}^{+} \\
& \equiv \quad R_{\alpha_{1}}^{+} f \cdot\left[e_{\alpha_{1}}^{-}, e_{\alpha_{2}}^{+} \ldots e_{\alpha_{k}}^{+}\right] \\
& =\quad-R_{\alpha_{1}}^{+} f \cdot\left[e_{\alpha_{1}}^{-}, e_{\alpha_{2}}^{+}\right] e_{\alpha_{3}}^{+} \ldots e_{\alpha_{k}}^{+} \\
& -R_{\alpha_{1}}^{+} f e_{\alpha_{2}}^{+}\left[e_{\alpha_{1}}^{-}, e_{\alpha_{3}}^{+}\right] \ldots e_{\alpha_{n}}^{+}+\ldots \in \mathfrak{g}_{\overline{0}}^{-} U\left(\mathfrak{g}_{\overline{0}}\right) \mathfrak{g}_{\overline{0}}^{-}
\end{aligned}
$$

\subsection{Proof of Lemma 1.2.1. Set}

$$
\Delta_{i}^{(k)+}=\tilde{\partial}_{i}^{(k)}+\tilde{\partial}_{\bar{i}}^{(k)}, \quad \Delta_{i}^{(k)-}=\tilde{\partial}_{i}^{(k)}-\tilde{\partial}_{\bar{i}}^{(k)} .
$$

Now, it is not difficult to verify the following identities:

$$
\begin{gathered}
\Delta_{i}^{(k)+}-\Delta_{i}^{(k-1)+}=\partial_{i} \Delta_{i}^{(k-1)-}+\sum_{j \neq i} \frac{2}{e^{\varepsilon_{i j}}-e^{\varepsilon_{j i}}}\left(\Delta_{i}^{(k-1)-}-\Delta_{j}^{(k-1)-}\right), \\
\Delta_{i}^{(k)-}=\left(\partial_{i}-1\right) \Delta_{i}^{(k-1)+}+\sum\left(\frac{2}{e^{\varepsilon_{i j}}-e^{\varepsilon_{j i}}} \Delta_{i}^{(k-1)+}-\frac{2 e^{\varepsilon_{i j}}}{e^{\varepsilon_{i j}}-e^{\varepsilon_{j i}}} \Delta_{j}^{(k-1)+}\right) .
\end{gathered}
$$

Further, if $\Delta_{i}^{(2 k)+}=f_{2 k}\left(\partial_{i}^{(1)}, \ldots, \partial_{i}^{(2 k-1)}\right)$ and $\Delta_{i}^{(2 k+1)^{+}}=f_{2 k+1}\left(\partial_{i}^{(1)}, \ldots, \partial_{i}^{(2 k+1)}\right)$, where $f_{2 k}$ and $f_{2 k+1}$ are any linear functions, then

$$
\begin{aligned}
f_{2 k+1} & =f_{2 k}+f_{2 k-1}\left(\partial_{i}^{(3)}, \ldots, \partial_{i}^{(2 k+1)}\right), \\
f_{2 k} & =f_{2 k-1}+f_{2 k-2}\left(\partial_{i}^{(3)}, \ldots, \partial_{i}^{(2 k-1)}\right) .
\end{aligned}
$$

It is easy to verify that

$$
\tilde{\Omega}_{1}=2 \Omega_{1}=\tilde{\Omega}_{2}, \quad \tilde{\Omega}_{3}=2 \Omega_{3}+2 \Omega_{1}, \quad \tilde{\Omega}_{4}=4 \Omega_{3}+2 \Omega_{1}
$$


and, by induction, (4.4.3) implies that $\tilde{\Omega}_{k}$ is a linear combination of the $\Omega_{2 l+1}$ for $2 l+1 \leq k$. One can show that, the other way round, $\Omega_{2 l+1}$ can be expressed via $\tilde{\Omega}_{k}$.

4.5. Proof of headings ii), iii) of Theorem 1.5. Statement of heading ii) is obvious. Heading iii) follows from Lemma 4.2 and the fact that $z_{k}=\sum_{i \in I} e_{i i}^{(k)}$.

4.6. Proof of heading iv) of Theorem 1.5. It is easy to verify that $\Theta^{*}=\sum e_{i}^{*} \otimes e_{i}^{*}$ is a $\mathfrak{p e}_{1}$-invariant whereas $\Theta=\sum_{i \in I} e_{i} \otimes e_{\bar{i}}$ is a $\mathfrak{p e}_{2}$-invariant. According to Se2 the linear hull of all the $\mathfrak{p} \mathfrak{e}_{1}$-invariants in $V^{\otimes 2 k}$ is isomorphic to

$$
\operatorname{Ind}_{H_{k}}^{\mathfrak{S}_{2 k}}(\varepsilon)=\underset{\lambda=\left(\alpha_{1}+1, \ldots, \alpha_{p}+1 \mid \alpha_{1}, \ldots, \alpha_{p}\right)}{\oplus} S^{\lambda}
$$

where $H_{k}=\mathfrak{S}_{k} \circ \mathbb{Z}_{2}^{k}$ is the semidirect product, and $h\left(\left(\Theta^{*}\right)^{\otimes k}\right)=\varepsilon(h)\left(\Theta^{*}\right)^{\otimes k}$ for any $h \in H_{k}$.

Similarly, the module of all the $\mathfrak{p e}_{2}$-invariants in $V^{\otimes 2 k}$ is of the form (4.6.1). This implies that

$$
\varphi_{\lambda}(u)=\Theta\left(v_{\lambda}^{*}, v_{\lambda}\right)(u)=\Theta\left(e_{\lambda} \Theta^{* 2 k}, e_{\lambda} \Theta^{2 k}\right)(u),
$$

where $e_{\lambda}$ is the minimal idempotent in the Hecke algebra $H\left(\mathfrak{S}_{2 k}, H_{2 k}, \varepsilon\right)$ corresponding to partition $\lambda$, see $\| S t]$.

If $\sigma \in \mathfrak{S}_{2 k}$, then the map

$$
\sigma \mapsto \varphi_{\sigma}, \text { where } \varphi_{\sigma}(u)=(-1)^{\frac{1}{2} k(k-1)} \Theta^{* \otimes k}\left(\sigma u \Theta^{\otimes k}\right)
$$

satisfies

$$
\varphi_{h_{1} \sigma h_{2}}=\varepsilon\left(h_{1} h_{2}\right) \varphi_{\sigma} \text { for any } h_{1}, h_{2} \in H_{k} .
$$

Therefore, we obtain a map $H\left(\mathfrak{S}_{2 k}, H_{k}, \varepsilon\right) \longrightarrow U(\mathfrak{g})^{* i n v}$. By restricting this map onto $S\left(\mathfrak{h}_{\overline{0}}\right)$ we obtain a map

$$
\operatorname{ch}: H\left(\mathfrak{S}_{2 k}, H_{k}, \varepsilon\right) \longrightarrow S\left(\mathfrak{h}_{\overline{0}}\right)^{*} .
$$

Let $K=K_{1} \cup K_{2}$, where $K_{1}=\left\{1, \ldots, 2 k_{1}\right\}, \quad K_{2}=\left\{2 k_{1}+1, \ldots, 2 k_{1}+2 k_{2}\right\}$ and let $\sigma_{1}$ permute the elements of $K_{1}$ whereas $\sigma_{2}$ permutes the elements of $K_{2}$; let $\sigma=\sigma_{1} \sigma_{2}$. Now, one can verify that

$$
\operatorname{ch}\left(\sigma_{1} \sigma_{2}\right)=\operatorname{ch}\left(\sigma_{1}\right) \operatorname{ch}\left(\sigma_{2}\right)
$$

Let now $k$ be odd and $\sigma=(1, \ldots, 2 k)$ a cycle. Let us calculate $\operatorname{ch}(\sigma)$. We have

$$
\Theta^{\otimes k}=\sum e_{\psi}, \quad \Theta^{* \otimes k}=\sum e_{\psi}^{*}
$$

where the sums run over all the maps $\psi:\{1, \ldots, 2 k\} \mapsto\{1, \ldots, n, \overline{1}, \ldots, \bar{n}\}$ such that $\psi(2 i)=\overline{\psi(2 i-1)}$ for $i=1, \ldots, k$ and where $e_{\psi}=e_{\psi_{(1)}} \otimes \ldots \otimes e_{\psi_{(2 k)}}$ and $e_{\psi}^{*}$ is similarly defined. Therefore,

$$
\operatorname{ch}(\sigma)(u)=(-1)^{\frac{1}{2} k(k-1)} \Theta^{* \otimes k}\left(u \sum \sigma e_{\psi}\right),
$$

where in the sum one has to take into account only the summands $e_{\psi}$ for which $\sigma \psi$ possesses the same property as $\psi$ does, i.e., $\psi(2 i)=\overline{\psi(2 i-1)}$. Therefore, we may assume that

$$
\psi(1)=\overline{\psi(2)}=\psi(3)=\overline{\psi(4)}=\ldots=\psi(2 k-1)=\overline{\psi(2 k)} .
$$


i.e., $e_{\psi}=\left(e_{i} \otimes e_{\bar{i}}\right)^{\otimes k}$ for $i \in\{1, \ldots, n, \overline{1}, \ldots, \bar{n}\}$. But for such $\psi$ and $u \in S\left(\mathfrak{h}_{\overline{0}}\right)$ we have

$$
\begin{aligned}
\operatorname{ch}(\sigma)(u) & =(-1)^{\frac{1}{2} k(k-1)} \Theta^{* \otimes k}\left(u \sum_{i \in I} \sigma\left(e_{i} \otimes e_{\bar{i}}\right)^{\otimes k}\right) \\
& =(-1)^{\frac{1}{2} k(k-1)} \Theta^{* \otimes k}\left(u \sum_{i \in I}\left(e_{i} \otimes e_{\bar{i}}\right)^{\otimes k}\right) \\
& =(-1)^{\frac{1}{2} k(k-1)} \Theta^{* \otimes k}\left(\sum_{i \in I_{\overline{0}}} 2 e^{\varepsilon_{i}}(u)\left(e_{i} \otimes e_{\bar{i}}\right)^{\otimes k}\right) \\
& =2 \sum_{i \in I_{\overline{0}}} 2 e^{k \varepsilon_{i}}(u) .
\end{aligned}
$$

Hence, $\operatorname{ch}(\sigma)=2 \sum_{i=1}^{n} 2 e^{k \varepsilon_{i}}$. Therefore, if $\sigma=\sigma_{\nu_{1}} \ldots \sigma_{\nu_{p}}$ is the product of independent cycles of odd lengths, then

$$
\operatorname{ch}(\sigma)=2^{l(\nu)} P_{\nu}, \text { where } P_{\nu}=P_{\nu_{1}} \ldots P_{\nu_{p}} \text { and } P_{l}=\sum_{i=1}^{n} e^{l \varepsilon_{i}} .
$$

We see that the map (4.6.2) coincides with the characteristic map Stembridge constructed in [St], p. 85. Therefore, by Theorem 5.2 from [St] we have

$$
\operatorname{ch}\left(e_{\lambda}\right)=\Theta_{\lambda}\left(e^{\varepsilon_{1}}, \ldots, e^{\varepsilon_{n}}\right) \cdot 2^{n-e(\lambda)} \cdot g_{\lambda},
$$

where $g_{\lambda}$ is the number of shifted standard tableaux of shape $\lambda$.

\section{REFERENCES}

[Dix] Dixmier J., Enveloping algebras. Revised reprint of the 1977 translation. Graduate Studies in Mathematics, 11. American Mathematical Society, Providence, RI, 1996. xx+379 pp.

[G] Gorelik M., Annihilation theorem and separation theorem for basic classical Lie superalgebras, math/RT0008143; Gorelik M., Lanzmann E., The annihilation theorem for the completely reducible Lie superalgebras. Invent. Math. 137 (1999), no. 3, 651-680

[Ma] Macdonald I.,Symmetric functions and Hall polynomials. With contributions by A. Zelevinsky. Oxford Mathematical Monographs. Oxford Science Publications. The Clarendon Press, Oxford University Press, New York, 1995. x+475 pp.

[Pe] Penkov I., Characters of typical irreducible finite-dimensional $\mathfrak{q}(n)$-modules. Funktsional. Anal. i Prilozhen. 20 (1986), no. 1, 37-45, 96 (Russian; English translation: Functional Anal. Appl. 20 (1986), no. 1, 30-37); Penkov I., Serganova, Vera Characters of finite-dimensional irreducible $\mathfrak{q}(n)$ modules. Lett. Math. Phys. 40 (1997), no. 2, 147-158; id., Characters of irreducible $G$-modules and cohomology of $G / P$ for the Lie supergroup $G=Q(N)$. Algebraic geometry, 7. J. Math. Sci. (New York) 84 (1997), no. 5, 1382-1412; Penkov I., Characters of strongly generic irreducible Lie superalgebra representations. Internat. J. Math. 9 (1998), no. 3, 331-366

[Sch] Schur I., Über die darstellung der symmetrischen und der alternierden Grouppe durch gebrochene lineare Substitutionen. J. Rein. Angew. Math., v. 139, 1911, 155-250

[Se1] Sergeev A., The tensor algebra of the identity representation as a module over Lie superalgebras $\mathfrak{g l}(m \mid n)$ and $\mathfrak{q}(n)$. Math. USSR Sbornik, v. 51, 1985, 419-427

[Se2] Sergeev A., An analog of the classical invariant theory for Lie superalgebras. I, II. mathRT/9810111, 9904079; Michigan J. Math., to appear

[Se3] Sergeev A., The centre of enveloping algebra for the Lie superalgebra $\mathfrak{q}(n ; \mathbb{C})$. Lett. Math. Phys. v. 7,1983 , no. $3,177-179$

[Se4] Sergeev A., The invariant polynomials on simple Lie superalgebras. Representation Theory, v. 3, 1999, $250-280$

[St] Stembridge J., On Schur's $Q$-functions and the primitive idempotents of a commutative Hecke algebra, J. Alg. Combinatorics, v. 1, 1992, no. 1, 71-95

Balakovo Institute of Technique, Technology and Control,Chapaeva 140 , Balakovo, Saratov Region, Russia

E-mail address: sergeev@bittu.org.ru 